\title{
Unexpected Visual Loss in Appropriately Treated Giant Cell Arteritis
}

\author{
Hannah Burton, MD candidate (2017), Laura Farrell, MD FRCPC MMed, Edward J Atkins, MD FRCPC
}

\section{About the Authors}

Hannah Burton psychiatry resident, graduate from the UBC Island Medical Program class of 2017. Laura Farrell, is the assistant dean of the Island Medical Program at the University of British Colombia. Edward J Atkins is an assistant clinical professor at the University of British Columbia (Medicine) in the Divisions of Neurology and Ophthalmology.

Correspondence may be directed to: hmburton@alumni.ubc.ca

Submitted: January 11, 2017. Accepted: July 31, 2017. November 7, 2017.

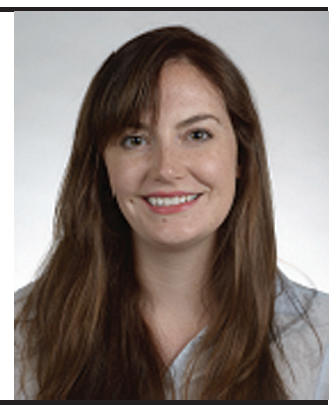

\begin{abstract}
This is a case report of a 92-year-old woman with Giant Cell Arteritis (GCA). After difficulties with diagnosis causing a delay in treatment, the patient was started on $1 \mathrm{mg} / \mathrm{kg} /$ day of oral prednisone, as recommended by most guidelines. Her symptoms improved initially but on day 7 of treatment she suddenly lost vision in her left eye and was diagnosed with Arteritic Anterior Ischemic Optic Neuropathy (AAION) in the setting of GCA. We provide an up to date literature review of the diagnosis, management, and visual deterioration in treated GCA, and discuss the importance of early treatment in this condition.
\end{abstract}

\section{Résumé}

Nous présentons un cas d'artérite temporale (AT) chez une femme âgée de 92 ans. Après quelques difficultés rencontrées dans létablissement d'un diagnostic et un délai à entreprendre un traitement, la patiente fut mise sous prednisone per os, $1 \mathrm{mg} / \mathrm{kg} /$ jour, comme le recommandent la plupart des lignes directrices. Les symptômes se sont tout d’abord améliorés, mais au jour 7 du traitement la patiente perdit soudainement la vue de l’eil gauche. On diagnostiqua alors une neuropathie optique ischémique antérieure artéritique (NOIAA) dans le contexte de l'AT. Nous présentons une revue récente de la littérature sur le diagnostic, la prise en charge et l'affaiblissement de la vue dans le contexte d'une AT sous traitement et nous discutons de l'importance d'un diagnostic précoce dans un tel cas.

A 92-year-old woman presented to the emergency department with a 2-week history of a progressive and persistent generalized headache. She denied neck stiffness, recent visual changes, jaw claudication, facial pain, anorexia, weight loss, fever, myalgia, or arthralgia. Contributory past history included dry Age Related Macular Degeneration (ARMD), recurrent urinary tract infections (UTIs), anxiety, and depression. Cardiovascular, respiratory, abdominal and neurologic exam were normal. She had baseline visual acuity of 20/50 in both eyes, and her pupils were equal and reactive to light without signs of Relative Afferent Pupillary Defect (RAPD). Initial white blood cell count was $13.4 \times 10^{9} / \mathrm{L}$, hemoglobin was $127 \mathrm{~g} / \mathrm{L}$ and platelets were $260 / \mathrm{L}$. Sodium was $132 \mathrm{mmol} / \mathrm{L}$, and potassium at $3.0 \mathrm{mmol} / \mathrm{L}$. Creatinine,
International Normalized Ratio, and liver enzymes were normal. C-reactive protein (CRP) was markedly elevated at $152.2 \mathrm{mg} / \mathrm{L}$.

She was admitted to the Internal Medicine Clinical Teaching Unit as she was unable to cope at home. The differential for her headache included tension headaches and Giant Cell Arteritis (GCA). The patient's inflammatory and infectious work-up was unremarkable. Malignancy work-up was also negative aside from her known invasive ductal carcinoma localized to the left breast. She was awaiting excisional surgery for invasive ductal carcinoma of the left breast recently diagnosed on biopsy and imaging showed no signs of metastatic disease.

On day 2 of admission, a trial of oral prednisone at $1 \mathrm{mg} / \mathrm{kg}$ daily was started for possible GCA with $81 \mathrm{mg}$ of acetylsalicylic 
acid (ASA) and general surgery was consulted regarding a temporal artery biopsy. After two days of therapy, the patient denied improvement in her symptoms. The team was concerned about the sequelae of committing this patient to steroids given her age, suspected cognitive impairment, and comorbidities, and therefore discontinued the steroids at that time. The patient's headache reportedly resolved with tylenol with codeine, providing support for the diagnosis of tension-type headache. The CRP had decreased by half and it was felt that the elevation may be due to her known breast cancer, or an UTI.

Several additional factors complicated this patient's admission. She experienced multiple falls in hospital, received a score of 11/30 on the Montreal Cognitive Assessment (MOCA) test, and endorsed symptoms of both anxiety and depression, causing the patient's hospital stay to be lengthened to set up daily home supports and to connect her with geriatric and psychiatric follow up. After 8 days without endorsing any headache symptoms and on day 12 as an inpatient, the patient described an escalating headache and proximal leg pain. She denied any visual symptoms, but a repeat CRP was $162 \mathrm{mg} / \mathrm{L}$. This resurgence of symptoms prompted a second trial of 1 $\mathrm{mg} / \mathrm{kg} /$ day prednisone along with $81 \mathrm{mg}$ ASA, and an urgent ophthalmology consult. Indirect ophthalmoscopy was notable for the known ARMD, but there was no disc edema, pallor, or blurred disc margins to suggest Arteritic Anterior Ischemic Optic Neuropathy (AAION). Temporal artery ultrasound was negative for a halo sign and a temporal artery biopsy was again requested. After two days of her second prednisone trial, her headache and leg pain resolved. Despite symptom resolution, the team monitored the patient in hospital for her prednisone treatment as she was deconditioned and had heightened anxiety on the high dose steroids resulting in concerns about her ability to cope at home. Unfortunately, after 7 days in hospital without symptoms on the second prednisone trial, the patient reported sudden left sided visual loss. Examination was notable for a new RAPD and decreased visual acuity to No Light Perception in the left eye and 20/60 in the right eye. Immediate ophthalmologic exam confirmed pallid disc edema of both eyes, most severe in the left eye, consistent with AAION in the setting of presumed GCA. Fluorescein angiography (FA) confirmed disc edema in

Table 1. American College of Rheumatology Diagnostic Criteria for GCA

Presence of 3 or more of the following criteria:

- greater than or equal to 50 years at disease onset

- new onset of localized headache

- temporal artery tenderness or decreased temporal artery pulse

- elevated ESR or CRP

- biopsy sample including an artery showing necrotizing arteritis the left eye greater than the right eye. Methylprednisolone $1 \mathrm{~g}$ intravenous (IV) was administered for three days, and then oral prednisone at a dose of $80 \mathrm{mg}$ was resumed.

After one day of IV methylprednisolone, her vision in the left eye improved. Her final visual outcome was $20 / 60$ in the right eye and 20/50 in the left eye (consistent with pre-existing ARMD). She was transitioned to oral prednisone and eventually discharged home with supports.

\section{Discussion}

\section{Diagnosis}

\section{Typical Presentation}

GCA, as defined by the American College of Rheumatology, is a large and medium-sized vessel vasculitis affecting individuals over the age of 50 that typically presents as new localized headache, temporal artery tenderness, elevated CRP or erythrocyte sedimentation rate and a positive temporal artery biopsy (Table 1). ${ }^{1}$ Symptoms of diplopia and jaw claudication, and exam findings of beading or prominent temporal arteries have the highest likelihood ratios suggesting GCA. ${ }^{2}$ Approximately $40-60 \%$ of patients with GCA also have symptoms of Polymyalgia Rheumatica (PMR), manifesting as pain and stiffness in the shoulder and pelvic girdle, proximal arms, and neck. ${ }^{3}$

\section{Difficulties with Diagnosis}

Despite these diagnostic parameters, up to $40 \%$ of patients with true GCA present atypically, ${ }^{4}$ and $25 \%$ of patients present with visual loss alone. ${ }^{5}$ Since visual loss can be prevented with treatment, correct diagnosis of GCA is exceptionally important.

GCA increases in prevalence with age, which unfortunately places many of the patients at risk of GCA also at risk for neurocognitive deficits. The difficulties with diagnosing medical ailments in patients with dementia or mental illness is well known due to the patients' decreased ability to detect and disclose physical symptoms. As a result, a clinician must find a balance between treating symptoms that may be a result of mental health or neurocognitive deficits too liberally or disregarding symptoms that could be a result of physical pathology. ${ }^{6}$ In our case, the patient met criteria for the diagnosis of GCA at admission; however, the description of her symptoms was vague and the symptoms fluctuated possibly due to an underlying neurocognitive disorder, resulting in a delay in diagnosis and appropriate treatment. This demonstrates the difficulties that may be encountered in the diagnosis of GCA in the geriatric population, and reinforces the need to pursue confirmatory testing especially if the diagnosis is unclear. 


\section{Confirming the Diagnosis}

The gold standard of GCA diagnosis is a positive temporal artery biopsy. Typical lesions on GCA positive biopsy include inflammatory cells within all layers of the arterial wall and changes to the inner elastic lamina ${ }^{7}$. Though a positive temporal artery biopsy has a specificity of $100 \%$, normal areas found between pathologic regions, referred to as "skip lesions," mean the false negative can be variable. As a result, the sensitivity has been previously reported from $7 \%$ to as high as $44 \%{ }^{8}$

As a result, the diagnosis of GCA is often made on clinical grounds based on steroid-responsiveness; however, the length of time required for symptoms to resolve after starting steroids has not been clearly defined. Some sources express that there should be a rapid resolution of symptoms, and that an absence of clinical improvement suggests considering alternative diagnoses. ${ }^{9}$

The patient was restarted on steroids as her headache symptoms had returned and she had developed symptoms of PMR. A temporal artery ultrasound was negative while awaiting biopsy. This test has a sensitivity of $68 \%$ and a specificity of $91 \%$, thus we could not rule out GCA. ${ }^{3}$ New imaging techniques such as 18 fluorodeoxyglucose-positron emission tomography (FDG-PET) have been proven to detect large vessel involvement in GCA, ${ }^{3}$ but FDG-PET is not available at our site. Magnetic resonance imaging/angiography (MRI/MRA) has also been shown to have a higher sensitivity and specificity and may have aided in diagnosing our patient. ${ }^{3}$

In the end, once the ophthalmologic sequelae of GCA was confirmed by fluoroangiography (FA), performing a temporal artery biopsy was unlikely to change diagnosis or management and this procedure was cancelled.

\section{Visual Loss Associated with GCA}

Permanent visual loss occurs in approximately $10 \%$ of GCA patients. ${ }^{5}$ The most common cause of visual loss in GCA is AAION, or vascular insufficiency to the anterior optic nerve. ${ }^{5}$ Funduscopy in AAION demonstrates immediate pallid disc edema after visual loss. ${ }^{5}$ Our patient's ophthalmologic findings on examination were consistent with AAION and confirmed GCA.

\section{Treatment}

Treatment of GCA without visual complications with $1 \mathrm{mg} / \mathrm{kg}$ per day of oral prednisone for several weeks followed by a slow taper has been well established in the literature. ${ }^{3}$ Since ASA is generally well tolerated and decreases inflammation and platelet aggregation within the vessel wall, it is thought that ASA may be a useful adjunct in the treatment of GCA; however, randomized control trials have not been completed on this topic (Table 2 and Table 3$).{ }^{10}$
Very recent studies have found varicella zoster virus (VZV) present within both GCA-positive and GCA-negative temporal artery biopsies within the skip lesions neighbouring areas of pathology, suggesting the possibility that VZV may play a critical role in triggering GCA. ${ }^{11}$ It has been hypothesized that adding antivirals to the above regimen may improve outcomes, although no trials have been completed to date.

\section{Treatment of Visual Loss}

When visual loss due to GCA occurs, commonly recommended treatment is $1 \mathrm{~g}$ of IV methylprednisolone for one to three doses 24 hours apart, followed with oral prednisone at $1 \mathrm{mg} / \mathrm{kg}$ per day for at least two weeks prior to beginning a slow taper. ${ }^{12}$ Some sources in the literature even suggest that three treatments of high dose oral steroids are sufficient initially before stepping down to the $1 \mathrm{mg} / \mathrm{kg}$ per day oral prednisone regimen, ${ }^{9}$ and this topic continues to be debated. As our patient was already admitted to hospital and her vision deteriorated after a week of oral steroid therapy, she was given IV steroids for three consecutive days. Fortunately, her vision improved within 24 hours.

A retrospective study done by Loddenkemper et al. in 2007 compared 14 cases of GCA with visual deterioration despite high-dose steroid therapy. ${ }^{13}$ Patients were treated differently based on physician preference and location, and results from patients treated with IV methylprednisolone initially were compared with patients started on oral high dose prednisone. Regardless of this difference, most patients experienced visual loss within the first 3 days of starting steroid treatment, which was pathologically determined to be a progression of vision changes that had begun prior to therapy. The remainder of patients experienced visual loss during the steroid taper many months later. The risk factors for visual loss after beginning steroid treatment were female sex, older age, worse initial visual

Table 2. Risk Factors for Visual Deterioration in GCA Prior to Starting Treatment

\section{Risk Factors}

\section{- optic disc swelling}

- history of hypertension

- older age

Table 3. Risk Factors for Visual Deterioration in GCA Despite Treatment

\begin{tabular}{|l|}
\multicolumn{1}{c|}{ Risk Factors } \\
\hline - female sex \\
\hline - older age \\
\hline - worse initial visual acuity \\
\hline - initial oral steroid treatment \\
\hline - higher ESR/CRP \\
\hline
\end{tabular}


acuity, higher ESR/CRP, and starting $1 \mathrm{mg} / \mathrm{kg}$ oral prednisone rather than starting high dose IV methylprednisolone.13 Our patient was treated with the standard oral steroid dose for one week before developing symptoms of visual loss, suggesting that those at high risk for AAION may be at risk for a longer period of time after the initiation of standard oral prednisone treatment than is generally understood or anticipated.

\section{Conclusion}

Our case highlights both difficulties with the diagnosis and treatment of GCA in the geriatric population, which unfortunately resulted in a delay in optimal treatment for our patient. When the patient did commence the appropriate therapy, she had no visual impairment but developed visual loss due to AAION after seven days of standard $1 \mathrm{mg} / \mathrm{kg}$ oral prednisone, which is outside the trends of visual loss outlined in the previous literature. That being said, the patient did have all the aforementioned risk factors associated with early visual loss on oral prednisone treatment. ${ }^{13}$

Fortunately, aggressive high dose IV methylprednisolone therapy reversed vision loss caused by AAION.

More studies are needed to determine if adding antiviral therapy to the treatment regimen for GCA is warranted.

\section{Key Points}

- Only $60 \%$ of cases GCA present classically as new headache, temporal artery tenderness, and elevated CRP or ESR. Neurocognitive comorbidities in the geriatric population may result in a delay to diagnosis.

- Without adequate steroid dosing and treatment, a patient with GCA is at risk for permanent visual loss.

- Though rare, patients can experience visual loss within the first 3 days of commencement of oral steroids; however, our case suggests the risk period may be longer. Visual loss may also occur months later as oral steroids are weaned.

\section{References}

1. Hunder GG, Bloch DA, Michel BA, et al. The American College of Rheumatology 1990 criteria for the classification of giant cell arteritis. Arthritis Rheum 1990;33:1122-8.
2. Smetana J, Shmerling R. Does the patient have temporal arteritis. JAMA 2002 Jan;287(1):92-101.

3. Gonzalez-Gay MA, Pineiro A, G. Giant cell arteritis and polymyalgia rheumatica: an update. Curr Rheumatol Rep 2015 Feb;17(2):6.

4. Moltyaner Y, Tenenbaum J. Temporal arteritis: a review and case history. J Fam Pract 1996;43:294-300.

5. Biousse V, Newman NJ. Ischemic Optic Neuropathies. N Engl J Med 2015;372(24):1428-36.

6. Lyness JM, Bruce ML, Koenig HG, Parmelee PA, Schulz R, Lawton MP, Reynolds CF 3rd. Depression and medical illness in late life: report of a symposium. J Am Geriatr Soc 1996 Feb;44(2):198-203.

7. Zhou L, Luneau $\mathrm{K}$, et al. Clinicopathologic correlations in giant cell arteritis: A retrospective study of 107 cases. Ophthalmology 2009 Aug;116(8):1574-80.

8. Davies CG, May DJ. The role of temporal artery biopsies in giant cell arteritis. Ann R Coll Surg Engl 2011 Jan;93(1):4-5.

9. Dasgupta B, Borg FA, Hassan N, et al. BSR and BHPR guidelines for the management of giant cell arteritis. Rheumatology (Oxford) 2010;49:1594-7.

10. Mollan SP, Sharrack N, Burdon MA, Denniston AK. Aspirin as adjunctive treatment for giant cell arteritis. The Cochrane Collaboration 2014;(8).

11. Gilden D, Nagel MA. Varicella zoster virus and giant cell arteritis. Curr Opin Infect Dis 2016 Jun;29(3):275-9.

12. Hayreh SS, Biousse V. Treatment of acute visual loss in giant cell arteritis: should we prescribe high-dose intravenous steroids or just oral steroids? J Neuroophthalmol 2012 Sep;32(3):278-87.

13. Loddenkemper T, Sharma P, Katzan I, PLant GT. Risk factors for early visual deterioration in temporal arteritis. J Neurol Neurosurg Psychiatr 2007 Nov;78(11):1255-59.

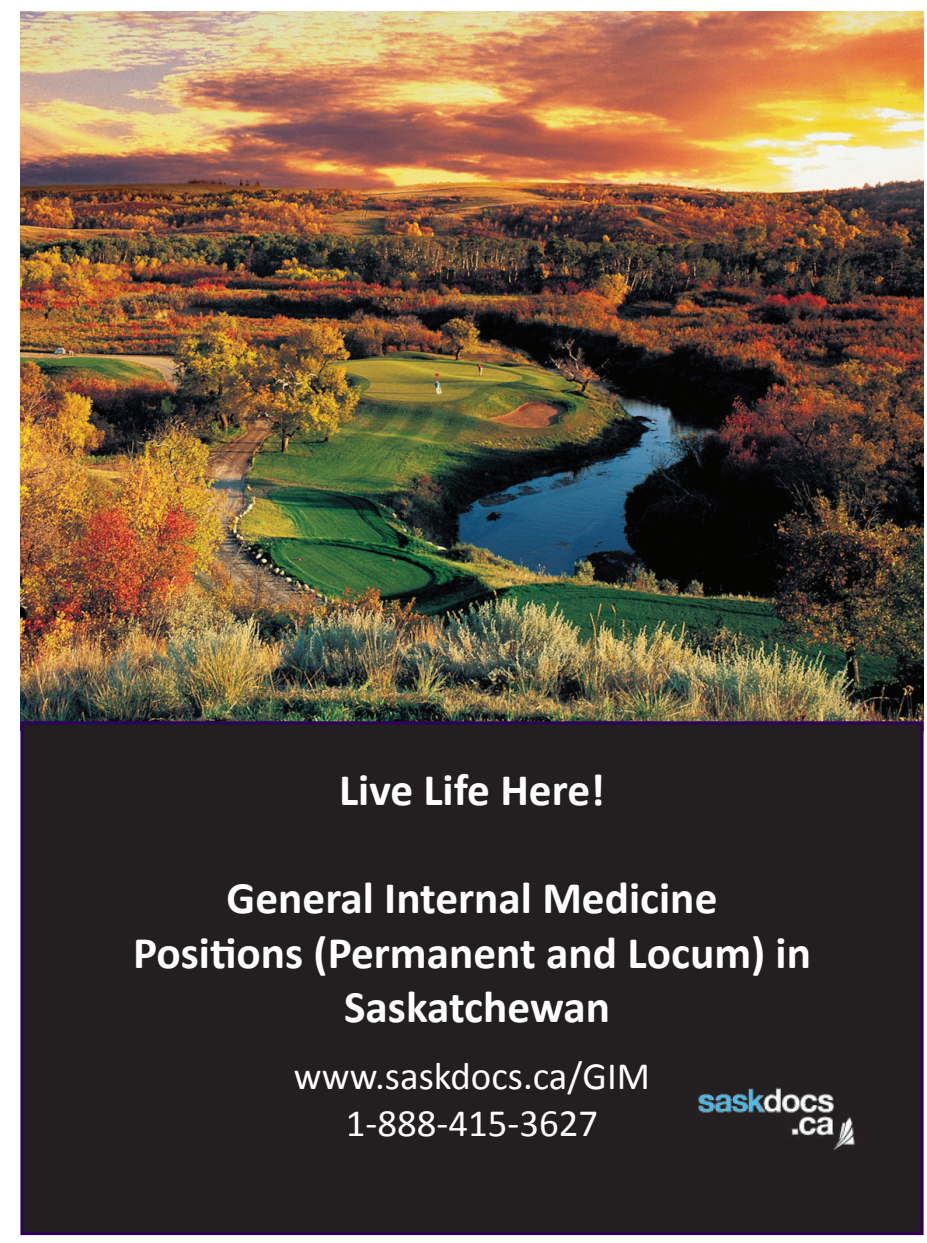

